\title{
CONTINUOUS FUNCTIONS WITH EVERYWHERE INFINITE VARIATION WITH RESPECT TO SEQUENCES
}

\author{
Z. BUCZOLICH
}

(Communicated by R. Daniel Mauldin)

ABSTRACT. We prove that if $\left\{a_{n}\right\}_{n=1}^{\infty}$ is such that $a_{n} \searrow 0$ and

$$
\lim _{n \rightarrow \infty}\left(a_{n+1} / a_{n}\right)=1 \text {, }
$$

then for the typical continuous function $f$ we have

$$
S_{n_{0}}:=\sum_{n=n_{0}}^{\infty}\left|f\left(x_{n+1}\right)-f\left(x_{n}\right)\right|=+\infty
$$

whenever $x \in\left[0,1-a_{n_{0}}\right]$ and $x_{n} \in\left[x+a_{n+1}, x+a_{n}\right]$.

Based on our result in a previous paper, we know that the above theorem fails to hold if $a_{n+1} / a_{n}=\lambda<1$.

We also prove that if $\left\{a_{n}\right\}_{n=1}^{\infty}$ is such that $a_{n} \searrow 0$, then for the typical continuous function $f$ we have $S_{n_{0}}=+\infty$ if $x_{n}=x+a_{n}$ and $x \in\left[0,1-a_{n_{0}}\right]$.

This paper is closely related to a recent study of intersections of typical continuous functions with certain classes of functions. The term "typical continuous function" means that the set of all functions which have the property under consideration is a residual subset of the complete metric space $C[0,1]$.

In $[\mathbf{H L}]$, P. Humke and M. Laczkovich proved that every continuous function agrees with an absolutely continuous function on a set which is not bilaterally strongly $x^{1+\delta}$ porous (Theorem 3). In fact, the authors proved that for any $f \in$ $C[0,1]$ and $\delta>0$ there is a point $x \in[0,1]$ and a sequence $x_{n} \rightarrow x$ such that $x_{n} \in\left[x+((n+1) !)^{-1-\delta}, x+(n !)^{-1-\delta}\right]$ and $\left|f\left(x_{n}\right)-f(x)\right|<n^{-1-\delta / 2}$ for $n>n_{0}$.

Let $g \in C[0,1]$ be linear on the intervals $[0, x],\left[x_{n+1}, x_{n}\right](n=1,2, \ldots),\left[x_{1}, 1\right]$, and agree with $f$ at the points $x_{n}$. Since $\sum_{n=n_{0}}^{\infty}\left|f\left(x_{n+1}\right)-f\left(x_{n}\right)\right|<+\infty, g$ is absolutely continuous and $f=g$ on a set which is not bilaterally strongly $x^{1+\delta}$ porous.

In [BU] we proved the following related result. For any Darboux function $f:[0,1] \rightarrow \mathbf{R}$ and any $0<\delta<1$, there is a point $x \in[0,1-\delta]$ and a sequence $x_{n}$ such that $x_{n} \in\left[x+\delta^{n+1}, x+\delta^{n}\right](n=1,2, \ldots)$ and $\sum_{n=1}^{\infty}\left|f\left(x_{n+1}\right)-f\left(x_{n}\right)\right|<+\infty$.

This theorem with a function $g$ defined similarly to the foregoing definition yields the following corollary:

For every $f \in C[0,1]$ there is an absolutely continuous function $g$ such that $\{x: f(x)=g(x)\}$ is not bilaterally strongly porous.

The last exponential type restriction on $x_{n}$ seems to be the strongest for which we can obtain a theorem of the above type. In this paper we prove the following theorem.

Received by the editors March 10, 1987.

1980 Mathematics Subject Classification (1985 Revision). Primary 26A15. 
THEOREM 1. Let $\left\{a_{n}\right\}_{n=1}^{\infty}$ be a sequence such that $a_{n} \searrow 0$ and

$$
\lim _{n \rightarrow \infty}\left(a_{n+1} / a_{n}\right)=1
$$

then there exists $f \in C[0,1]$ such that, for all $s \in \mathbf{N}, x \in\left[0,1-a_{s}\right]$ and for all sequences $\left\{x_{n}\right\}, x_{n} \in\left[x+a_{n+1}, x+a_{n}\right]$, we have

$$
\sum_{n=s}^{\infty}\left|f\left(x_{n+1}\right)-f\left(x_{n}\right)\right|=+\infty \text {. }
$$

Furthermore, the set of the functions $f$ having the above property is dense $G_{\delta}$; that is, the typical continuous function has this property.

If we choose to let $x_{n}=x+a_{n}$, then the restriction that $\lim _{n \rightarrow \infty}\left(a_{n+1} / a_{n}\right)=1$ may be deleted as follows:

THEOREM 2. Let $\left\{a_{n}\right\}_{n=1}^{\infty}$ be a sequence with $a_{n} \searrow 0$; then for any $s \in \mathbf{N}$, $x \in\left[0,1-a_{s}\right]$, we have $\sum_{n=s}^{\infty}\left|f\left(x+a_{n+1}\right)-f\left(x+a_{n}\right)\right|=+\infty$ for the typical continuous function $f \in C[0,1]$.

LEMMA 1. Suppose that we have a sequence $\left\{a_{n}\right\}_{n=1}^{\infty}$ such that $a_{n} \searrow 0$ and $\lim _{n \rightarrow \infty}\left(a_{n+1} / a_{n}\right)=1$. Furthermore, let $K \in \mathbf{N}$ and $M_{1} \in \mathbf{N}$. Then there exists $g \in C(\mathbf{R})$ and $M_{2} \in \mathbf{N}$ such that

(1) $\sup _{x \in \mathbf{R}}|g(x)|=1 / K$,

(2) for all $x \in \mathbf{R}$ and $\left\{x_{n}\right\}_{n=1}^{\infty}, x_{n} \in\left[x+a_{n+1}, x+a_{n}\right]$, we have

$$
\sum_{n=M_{1}}^{M_{2}-1}\left|g\left(x_{n+1}\right)-g\left(x_{n}\right)\right|>1 \text {, }
$$

(3) $g$ is periodic and piecewise linear.

ProOF. First we choose an $n_{0} \geq M_{1}$ such that

$$
1-a_{i+1} / a_{i}<1 /(16 \cdot K) \text { for } i \geq n_{0}
$$

and let

$$
d:=a_{n_{0}} / 8 K
$$

We define the function $g \in C(\mathbf{R})$ by

$$
g(x):= \begin{cases}0 & \text { if } x \in[4 k \cdot d,(4 k+1) \cdot d], k \in \mathbf{Z}, \\ 1 / K & \text { if } x \in[(4 k+2) \cdot d,(4 k+3) \cdot d], k \in \mathbf{Z} \text { and } g \text { is linear } \\ & \text { on the intervals contiguous to the above intervals. }\end{cases}
$$

Obviously, (1) and (3) are fulfilled. Let $x \in \mathbf{R}$ be given and $x \in[l \cdot d,(l+1) \cdot d]$. We claim that for any $k \in\{(l+1), \ldots,(l+8 \cdot K-2)\}$ there is an $i_{k}$ such that

$$
\left[x+a_{i_{k}+1}, x+a_{i_{k}}\right] \subset[k \cdot d,(k+1) d] .
$$

Indeed, if $x+a_{j} \in[(l+1) d,(l+8 K-1) d]$, then $a_{j}<a_{n_{0}}$ and hence $a_{j-1} \leq a_{n_{0}}$. Consequently, by (4) and (5),

$$
x+a_{j-1}-\left(x+a_{j}\right)=a_{j-1}-a_{j}<\frac{1}{16 \cdot K} a_{j-1} \leq \frac{a_{n_{0}}}{16 \cdot K}=\frac{d}{2},
$$


and hence plainly, for any $k \in\{(l+1), \ldots,(l+8 K-2)\}$, there is an $i_{k}$ fulfilling (6). It follows that for $x_{i_{k}} \in\left[x+a_{i_{k}+1}, x+a_{i_{k}}\right] \subset[k \cdot d,(k+1) d]$ we have $g\left(x_{i_{k}}\right)=0$ if $4 \mid k$ and $g\left(x_{i_{k}}\right)=1 / K$ if $2 \mid k$ and $4 \nmid k$. We choose $M_{2}$ such that $a_{M_{2}}<d$ and we have

$$
\begin{aligned}
& \sum_{n=M_{1}}^{M_{2}-1}\left|g\left(x_{n+1}\right)-g\left(x_{n}\right)\right| \\
& \quad \geq \sum_{k^{\prime}=[(l+1) / 2]+1}^{[(l+8 K-2) / 2]}\left|\sum_{n=i_{2 k^{\prime}+2}}^{i_{2 k^{\prime}}-1} g\left(x_{n+1}\right)-g\left(x_{n}\right)\right| \\
& \quad=\sum_{k^{\prime}=[(l+1) / 2]+1}^{[(l+8 K-2) / 2]}\left|g\left(x_{i_{2 k^{\prime}}}\right)-g\left(x_{i_{2 k^{\prime}+2}}\right)\right|>\frac{1}{K} \cdot K=1 ;
\end{aligned}
$$

thus, (2) is also satisfied (where $[x]$ denotes the integer part of $x$ ).

PROOF OF THEOREM 1. First we prove the existence of an $f \in C[0,1]$ claimed in our theorem. We put $N_{0}=s, K_{1}=1$ and then, applying Lemma 1 with $K:=K_{1}$ and $M_{1}:=N_{0}$, we get $g_{1}$ and $N_{1}:=M_{2}$. Let $f_{1}:=\left.g_{1}\right|_{[0,1]}$. By (3), $f_{1}$ is a Lipschitz function and a fortiori a function of bounded variation $\left(f_{1} \in V B\right)$, and we denote its absolute variation by $V_{1}$. Suppose that we have defined $N_{l-1}, f_{l-1}, V_{l-1}$. Then we choose $K_{l}$ such that

$$
K_{l}>2^{l} \cdot N_{l-1} \cdot\left(V_{l-1}+3\right),
$$

and applying Lemma 1 with $K:=K_{l}$ and $M_{1}:=N_{l-1}$ we get $g_{l}$ and $N_{l}:=M_{2}$.

Let $f_{l}:=f_{l-1}+\left.\left(V_{l-1}+3\right) \cdot g_{l}\right|_{[0,1]}$. By (7) and (1) we have

$$
\sup _{x \in[0,1]}\left|f_{l}(x)-f_{l-1}(x)\right|<\frac{1}{2^{l} \cdot N_{l-1}} \text {. }
$$

It follows from (3) that $\left.g_{l}\right|_{[0,1]} \in V B$ and thus $f_{l} \in V B$, and we can define $V_{l}<\infty$ to be the absolute variation of $f_{l}$. It follows from (8) that there exists $\lim _{l \rightarrow \infty} f_{l}(x):=$ $f(x) \in C[0,1]$. We have, for any $x \in\left[0,1-a_{s}\right]$ and for all $\left\{x_{n}\right\}_{n=1}^{\infty}, x_{n} \in[x+$ $\left.a_{n+1}, x+a_{n}\right]$,

$$
\begin{aligned}
\sum_{n=N_{l-1}}^{N_{l}-1} & \left|f\left(x_{n+1}\right)-f\left(x_{n}\right)\right| \\
\geq & \sum_{n=N_{l-1}}^{N_{l}-1}\left(V_{l-1}+3\right)\left|g_{l}\left(x_{n+1}\right)-g_{l}\left(x_{n}\right)\right| \\
& \quad-\sum_{n=N_{l-1}}^{N_{l}-1}\left|f_{l-1}\left(x_{n+1}\right)-f_{l-1}\left(x_{n}\right)\right| \\
& \quad-\sum_{n=N_{l-1}}^{N_{l}-1} \sum_{k=l+1}^{\infty}\left(V_{k-1}+3\right)\left|g_{k}\left(x_{n+1}\right)-g_{k}\left(x_{n}\right)\right| \\
\geq & \left(V_{l-1}+3\right)-V_{l-1}-\sum_{k=l+1}^{\infty} \frac{2}{2^{k} N_{k-1}} \cdot N_{l}=: A_{l} .
\end{aligned}
$$


In the last estimation we used (2), the definition of the absolute variation, and (8). Since $N_{l} \leq N_{k-1}$ for $k \geq l+1$, we have $A_{l} \geq 1$ and hence

$$
\sum_{n=1}^{\infty}\left|f\left(x_{n+1}\right)-f\left(x_{n}\right)\right| \geq \sum_{l=1}^{\infty} A_{l}=+\infty
$$

Thus we proved that there exists at least one $f \in C[0,1]$ having the property of Theorem 1. We show that the sets

$$
\begin{array}{r}
G_{k}^{s}:=\left\{f \in C[0,1]: \sum_{n=s}^{\infty}\left|f\left(x_{n+1}\right)-f\left(x_{n}\right)\right|>k \text { for any } x \in\left[0,1-a_{s}\right]\right. \\
\text { and } \left.\left\{x_{n}\right\}_{n=1}^{\infty}, x_{n} \in\left[x+a_{n+1}, x+a_{n}\right]\right\}
\end{array}
$$

are dense open sets in $C[0,1]$. For any $f \in C[0,1]$, the function

$$
f_{m}(x):=\min \left\{\sum_{n=s}^{m}\left|f\left(x_{n+1}\right)-f\left(x_{n}\right)\right|: x_{n} \in\left[x+a_{n+1}, x+a_{n}\right]\right\}
$$

is continuous on $\left[0,1-a_{s}\right]$. Plainly $f_{m}(x) \leq f_{m+1}(x)$ for $m \geq s$, and for $f \in G_{k}^{s}$, $x \in\left[0,1-a_{s}\right]$ we have $\lim _{m \rightarrow \infty} f_{m}(x)>k$ and by the compactness of $\left[0,1-a_{s}\right]$ there exists an $m_{0}$ and $\varsigma>0$ such that $f_{m_{0}}(x)>k+\varsigma>k$ for any $x \in\left[0,1-a_{s}\right]$. Thus, taking any $g$ such that $\|g-f\|_{\max }<\varsigma / 2 m_{0}$, we have

$$
\left|\sum_{n=s}^{m_{0}}\right| f\left(x_{n+1}\right)-f\left(x_{n}\right)\left|-\sum_{n=s}^{m_{0}}\right| g\left(x_{n+1}\right)-g\left(x_{n}\right)||<\sum_{n=s}^{m_{0}} 2 \cdot \frac{\varsigma}{2 \cdot m_{0}} \leq \varsigma ;
$$

that is, $g_{m_{0}}(x)>f_{m_{0}}(x)-\varsigma>k$ and hence $g \in G_{k}^{s}$. Let $h \in C[0,1]$ and $\varepsilon>0$ be given. By the density of $C^{1}[0,1]$ in $C[0,1]$ there is $g \in C^{1}[0,1]$ such that $\|g-h\|_{\max }<\varepsilon / 2$ (where $C^{1}[0,1]:=\left\{f \in C[0,1]: f^{\prime} \in C[0,1]\right\}$ ). We have, by the first part of this proof, an $f \in C[0,1]$ fulfilling the property of Theorem 1 . Obviously, for any $\alpha \in \mathbf{R}, \alpha \cdot f$ also fulfills the property of Theorem 1, and taking $\alpha_{0}=\varepsilon / 2\|f\|_{\max }$ we have $\left\|\alpha_{0} \cdot f\right\|_{\max }<\varepsilon / 2$.

For any $\left\{x_{n}\right\}$ such that $x_{n} \in\left[x+a_{n+1}, x+a_{n}\right]$, we have

$$
\sum_{n=1}^{\infty}\left|g\left(x_{n+1}\right)-g\left(x_{n}\right)\right|=\sum_{n=1}^{\infty}\left|\int_{x_{n+1}}^{x_{n}} g^{\prime}(x) d x\right|<\int_{0}^{1}\left|g^{\prime}(x)\right| d x<+\infty
$$

and hence

$$
\begin{aligned}
+\infty & =\sum_{n=1}^{\infty}\left|\alpha_{0} f\left(x_{n+1}\right)-\alpha_{0} f\left(x_{n}\right)\right|-\sum_{n=1}^{\infty}\left|g\left(x_{n+1}\right)-g\left(x_{n}\right)\right| \\
& \leq \sum_{n=1}^{\infty}\left|\left(\alpha_{0} f+g\right)\left(x_{n+1}\right)-\left(\alpha_{0} f+g\right)\left(x_{n}\right)\right| .
\end{aligned}
$$

Since $\left\|h-\left(\alpha_{0} f+g\right)\right\|_{\max }<\varepsilon$, we prove that $G_{k}^{s}$ is a dense open set. Hence $\bigcap_{s, k=1}^{\infty} G_{k}^{s}$ is thus a dense $G_{\delta}$ and is therefore residual. 
LEMMA 2. If $0<a_{1}<\cdots<a_{k}$ and

$$
a_{i} \geq 3 \cdot k \cdot a_{i-1} \quad(i=2, \ldots, k),
$$

then there is $d \in\left[a_{1} / 2, a_{1}\right]$ such that

$$
\left[a_{i+1} / d\right]-\left[a_{i} / d\right] \text { is odd for } i=1, \ldots, k-1,
$$

and

$$
(i-1) / k<\left\{a_{i} / d\right\}<i / k \quad(i=1, \ldots, k), \text { where }\{x\}=x-[x] .
$$

PROOF. Let $b_{1}:=a_{1} /(1+(1 / k)), c_{1}:=a_{1}$, and $N_{1}:=1$. Then for every $d \in$ $\left(b_{1}, c_{1}\right)$ we have $\left[a_{1} / d\right]=1$ and $0<\left\{a_{1} / d\right\}<1 / k$. Suppose that $i \in\{1,2, \ldots, k-1\}$ and an integer $N_{i}>0$ is given. We put

$$
b_{i}:=\frac{a_{i}}{N_{i}+i / k}, \quad c_{i}:=\frac{a_{i}}{N_{i}+(i-1) / k} .
$$

Then

$$
a_{i+1} / b_{i}-a_{i+1} / c_{i}=a_{i+1} / a_{i} \cdot 1 / k \geq 3
$$

by (9), and hence there is an integer $N_{i+1}$ such that $2 \nmid N_{i+1}-N_{i}$ and

$$
\left[N_{i+1}, N_{i+1}+1\right] \subset\left[a_{i+1} / c_{i}, a_{i+1} / b_{i}\right] .
$$

Letting

we have by (12)

$$
b_{i+1}:=\frac{a_{i+1}}{N_{i+1}+(i+1) / k}, \quad c_{i+1}:=\frac{a_{i+1}}{N_{i+1}+i / k},
$$

$$
\frac{b_{i+1}}{b_{i}}=\frac{a_{i+1}}{b_{i}} \cdot \frac{1}{N_{i+1}+(i+1) / k} \geq \frac{a_{i+1}}{b_{i}} \cdot \frac{1}{N_{i+1}+1} \geq 1
$$

and

$$
\frac{c_{i+1}}{c_{i}}=\frac{a_{i+1}}{c_{i}} \cdot \frac{1}{N_{i+1}+i / k}<\frac{a_{i+1}}{c_{i}} \cdot \frac{1}{N_{i+1}} \leq 1 ;
$$

that is, $\left[b_{i+1}, c_{i+1}\right] \subset\left[b_{i}, c_{i}\right]$. For every $d \in\left(b_{i+1}, c_{i+1}\right)$ we obtain also $\left[a_{i+1} / d\right]=$ $N_{i+1}$ and $i / k<\left\{a_{i+1} / d\right\}<(i+1) / k$. In this way we get the intervals $\left[b_{1}, c_{1}\right] \supseteq$ $\cdots \supseteq\left[b_{k}, c_{k}\right]$ and, plainly, every $d \in\left(b_{k}, c_{k}\right)$ satisfies (10) and (11).

LEMMA $1^{\prime}$. Suppose that we have a sequence $\left\{a_{n}\right\}$ such that $a_{n} \searrow 0, K \in \mathbf{N}$ and $M_{1} \in \mathbf{N}$. Then there exists $g \in C(\mathbf{R})$ and $M_{2} \in \mathbf{N}$ such that

(13) $\sup _{x \in \mathbf{R}}|g(x)|=1 / K$,

(14) $\sum_{n=M_{1}}^{M_{2}-1}\left|g\left(x+a_{n+1}\right)-g\left(x+a_{n}\right)\right|>1$, and

(15) $g$ is periodic and piecewise linear.

PROOF. We put $k:=K+5$. Let $\left\{a_{n_{i}}\right\}_{i=1}^{k}$ be a subset of $\left\{a_{n}\right\}_{n=1}^{\infty}$ such that $n_{k}>M_{1}, a_{n_{1}}<\cdots<a_{n_{k}}$, and $a_{n_{i}} \geq 3 \cdot k \cdot a_{n_{i-1}}$ for $i=2, \ldots, k$. From Lemma 2 we get $d \in\left[a_{n_{1}} / 2, a_{n_{1}}\right]$ which fulfills (10) and (11). We define the continuous function $g$ by

$$
g(x):= \begin{cases}0 & \text { if } x \in[2 l \cdot d,(2 l+1) d-d / k], l \in \mathbf{Z} \\ 1 / K & \text { if } x \in[(2 l+1) d,(2 l+2) \cdot d-d / k], l \in \mathbf{Z} \text { and linear on } \\ & \text { the intervals contiguous to the above intervals. }\end{cases}
$$


By definition, $g$ fulfills (13) and (15). We put $M_{2}:=n_{1}$. Let $x \in \mathbf{R}$ be given. By (11) in Lemma 2 there are at most two subsequent $(\bmod k)$ indices $j, j+1$ such that $\left\{x+a_{n_{i}} / d\right\} \geq(k-1) / k$ (for $i=j$ or $j+1$ ); that is, $g\left(x+a_{n_{i}}\right)=0$ or $1 / K$ for $i \in\{1,2, \ldots, k\} \backslash\{j, j+1\}$. Furthermore, also by (11) we have

$$
\left[\frac{x+a_{n_{i+1}}}{d}\right]-\left[\frac{x+a_{n_{i}}}{d}\right]=\left[\frac{a_{n_{i+1}}}{d}\right]-\left[\frac{a_{n_{i}}}{d}\right]
$$

for $i \in\{1,2, \ldots, k\} \backslash\{j-1, j, j+1\}$. That is, by (10) and the definition of $g$, we have $\left|g\left(x+a_{n_{i+1}}\right)-g\left(x+a_{n_{i}}\right)\right|=1 / K$ for the above $i$ 's. Thus

$$
\begin{aligned}
\sum_{n=M_{1}}^{M_{2}-1}\left|g\left(x+a_{n+1}\right)-g\left(x+a_{n}\right)\right| & \geq \sum_{i=1}^{k-1}\left|\sum_{n=n_{i+1}}^{n_{i}-1} g\left(x+a_{n+1}\right)-g\left(x+a_{n}\right)\right| \\
& =\sum_{i=1}^{k-1}\left|g\left(x+a_{n_{i}}\right)-g\left(x+a_{n_{i+1}}\right)\right| \geq \frac{k-4}{K}>1,
\end{aligned}
$$

and this proves Lemma $1^{\prime}$.

PROOF OF THEOREM 2. Using Lemma $1^{\prime}$ instead of Lemma 1 we can repeat the proof of Theorem 1 .

\section{REFERENCES}

[BU] Z. Buczolich, For every continuous $f$ there is an absolutely continuous $g$ such that $[f=g]$ is not bilaterally strongly porous, Proc. Amer. Math. Soc. 100 (1987), 485-488.

[HL] P. Humke and M. Laczkovich, Typical continuous functions are virtually nonmonotone, Proc. Amer. Math. Soc. 94 (1985), 244-248.

Department of analysis, EÖtVös University, Budapest, MuZeum KRT. 6-8, H-1088, HUNGARY 